\title{
Management of patients with multiple myeloma in the era of COVID-19 pandemic: how hospital at home changes our medical practice
}

\author{
G. Fouquet $^{1}$ - P. Franchi ${ }^{1}$ - B. Mittaine-Marzac ${ }^{2,3} \cdot$ N. Laporte $^{1} \cdot$ H. Ihaddadene ${ }^{1} \cdot$ J. Decroocq $^{1} \cdot$ C. Breal ${ }^{1}$.

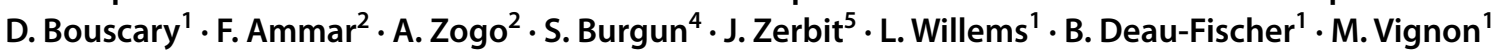

Received: 6 February 2021 / Accepted: 14 May 2021 / Published online: 31 May 2021

(c) The Author(s), under exclusive licence to Springer-Verlag GmbH Germany, part of Springer Nature 2021

\section{Background}

Oncology and hematology physicians aim during the COVID-19 pandemic to protect their patients from COVID-19 infection without impairing the prognosis of the disease. Consensus recommendations have thus been implemented for the management of cancer patients during this period [1]. Karacin et al. reported on the reorganization of oncology hospitals in Ankara [2] and described the increased use of telemedicine, admission procedures in "clean" and "pandemic" centers, and preventive strategies.

Patients with hematological diseases, especially multiple myeloma (MM), are at high risk of infection as they are often older than 65 years, and are highly immunosuppressed due to their treatments and the disease itself [2]. The management of patients with multiple myeloma is therefore a specific challenge in the context of COVID19 [3].

M. Vignon

marguerite.vignon@aphp.fr

1 Hematology Department, Cochin Hospital, APHP, Paris, France

2 Hospital At Home, APHP, Paris, France

3 CESP (Centre de Recherche en Epidémiologie Et Santé Des Populations), U1018 INSERM UPS Université Paris-Saclay, Villejuif, France

4 Hospitalization At Home, Fondation Santé Service, Paris, France

5 Pharmacy, Cochin Hospital, APHP, Paris, France

\section{Aims}

The challenge for clinicians is not only to adapt the treatment for $\mathrm{MM}$ to reduce immunosuppression but also to find innovative solutions to limit patients stay at hospital, and thereby minimize nosocomial transmission.

We describe how at-home treatment was used in our department to maintain multiple myeloma treatment while protecting the patient.

\section{Methods}

We describe the experience of our hematology department at Cochin, an academic hospital in the Ile-de-France region, which was deeply affected by the COVID-19 pandemic, during the first epidemic peak from March 20 to April 17, 2020 [4].

We compared this experience to previous months during a COVID-free period.

\section{Organization at our clinic during COVID-free period}

Our outpatient clinic receives about 140 patients per month, for multiple myeloma treatment: anti-CD38 monoclonal antibody $64 \%$, bortezomib $25 \%$, carfilzomib $10 \%$, and others $2 \%$ (doxorubicine, bendamustine). In most cases, those treatment are associated with oral chemotherapy (dexamethasone, IMID = lenalidomide and pomalidomide, alkylating agents). Patients usually collect their oral treatment at hospital when they come for their treatment injection.

We have been employing at-home chemotherapy protocols since 2010 [5]. Our "Hospital at Home" (HAH) program provides subcutaneous (SC) or intravenous (IV) chemotherapy at home for the patient. 
The antineoplastic agents must have sufficient stability, a short administration time (90 min maximum), and no risk of infusion-related reaction. Recently, we have also organized home infusions of the monoclonal antibodies daratumumab and isatuximab after several injections at hospital without any reactions [6].

Physicians prescribe chemotherapy from the hospital software, and chemotherapy is prepared in the pharmacy unit. Treatment is then delivered in a sealed temperaturecontrolled box by a secure carrier. After delivery, dedicated nurses go at home and administrate the treatment. Nurses are present during the injection. Then they validate the treatment injection in the software and inform the physician if there is any unexpected problem.

During our standard organization, patients eligible for $\mathrm{HAH}$ program come to the outpatient clinic for the first injection of cycle of the chemotherapy (e.g., for bortezomib d1). Medical validation is given for the other injection and the subsequent doses are delivered at home (e.g., bortezomib d8-d15-d22). Patients return to hospital for the beginning of the next cycle. Thus, HAH program avoids some routine hospital visits for anticancer drugs administration.

Eligibility criteria for our HAH program include the patient's consent and a stable housing situation in the Ilede-France region. Patients living alone may be eligible provided they are self-sufficient. A central venous catheter is not mandatory.

\section{Changes to clinical management protocols during the COVID-19 outbreak}

To avoid bringing immunocompromised and often elderly patients to our hospital during the COVID-19 outbreak, we greatly expanded our use of at-home treatments.

First, in accordance with the recommendations of cooperative groups like the European Myeloma Network [3], weekly meetings involving the physicians, a nurse, and a secretary were convened to decide which treatments to cancel.

Then, if treatments needed to be maintained, the appropriate place of administration was chosen: in our outpatient clinic, via the HAH program or at a local hospital. The following criteria were applied:
1. Treatments for patients showing good response to SC or IV maintenance therapy were cancelled. If an oral chemotherapy was associated, it was maintained and the required agents were sent by the hospital pharmacy for collection at a community pharmacy to reduce hospital visits.

2. Treatments were given at hospital in the outpatient clinic under the following circumstances: (1) a long parenteral treatment with multidrug therapy was required; (2) there was evidence of treatment toxicity or signs of disease relapse; (3) the patient reported symptoms to a nurse during a phone check-up prior to treatment that required a clinical examination.

3. All other cases were treated via the HAH program, or at another local hospital if they resided outside our prescribed $\mathrm{HAH}$ area.

\section{Results}

During the first epidemic peak from March 20 to April 17, 2020,143 patients at our clinic were scheduled to receive injected treatments for MM including SC bortezomib, IV carfilzomib, or IV daratumumab. However, 37 of these cases did not receive the initially planned treatment (Table 1) for the following reasons:

1. Seventeen patients had their SC or IV treatment cancelled. These therapies were cancelled directly in two cases because of the COVID-19 pandemic (infected 1, had travelled abroad 1). The other 15 patients had been showing a good response and their treatment was postponed.

2. Twenty patients continued to receive treatment outside the outpatient clinic. Fifteen of these cases received their treatment via the $\mathrm{HAH}$ program and were then also provided with concomitant oral medication. These cases received a monoclonal antibody (daratumumab $n=2$ ) or an IV or SC proteasome inhibitor (carfilzomib $n=4$, bortezomib $n=9$ ). Five patients living outside the Ile-de-France region could not access the HAH program and went to their local hospital.
Table 1 Management of multiple myeloma patients in the outpatient clinic during the initial peak (from March 20 to April 17 2020) of the COVID-19 pandemic $(n=143)$

\begin{tabular}{|c|c|c|c|c|c|}
\hline & & & Daratumumab & Bortezomib & Carfilzomib \\
\hline \multicolumn{6}{|c|}{ Patients treated at Cochin $n=106(75 \%)$} \\
\hline \multirow{3}{*}{$\begin{array}{l}\text { Change in treat- } \\
\text { ment plan } n=37\end{array}$} & Treatment cancelled & $n=17 *$ & $n=14$ & $n=2$ & $n=1$ \\
\hline & Home hospitalisation & $n=15$ & $n=2$ & $n=9$ & $n=4$ \\
\hline & $\begin{array}{l}\text { Treated at local } \\
\text { hospital }\end{array}$ & $n=5$ & $n=2$ & $n=2$ & $n=1$ \\
\hline
\end{tabular}

*oral treatment maintained in $14 / 17$ cases 
Each of these $37 \mathrm{MM}$ patients had tele-appointments with the physician to check their symptoms and blood test results, to ensure that they continued the appropriate oral treatment, and to schedule the next visit. Signs of relapse were carefully checked as well as signs of COVID-19 infection.

Overall, $25 \%$ of our MM patients had their treatment plan modified. We were thus able to reduce the number of patients at our outpatient clinic and help to prevent patient contamination at hospital. We did not see any unexpected relapses in these patients after 10 months of follow-up and patients were satisfied with this organization. Of note, one patient received also palliative care through the HAH program which enabled family interactions that would not have been possible in a palliative care unit.

During this period, HAH program was able to deal with an increased activity of $12 \%$ compared to the previous month.

\section{Discussion}

We have here described how the COVID-19 pandemic has modified the clinical practices at our hospital, with a particular focus on our MM patients. We wished to emphasize that the issue was not only to reduce immunosuppression but also to limit the time spent by these patients at hospital [7].

HAH program has already been proven cost-effective and preferred by the patients to hospital administration [8]. In the context of COVID-19 pandemic, administration of the MM treatments at home was safer than in a hospital setting.

HAH staff managed to ramp up its activity very quickly in these troubled time. It must be emphasized however that a successful at-home treatment protocol requires the full participation of the patient and every clinical staff member involved in providing this care including caregivers for patient navigation, administrative staff for reprogramming, physicians and nurses for treatment, and pharmacists for delivery of their required drugs to the local hospital or to the home.

The COVID-19 pandemic is still with us at the time of writing and HAH is currently helping us to prevent contamination for our patients. HAH programs should be considered a viable alternative to hospital-based treatments for certain cancer patients and can significantly reduce the hospital burden without compromising care. Unfortunately, its availability is unevenly distributed both in France and throughout the world [9]. At the end of the pandemic, however, the classical model of cancer management will change and home treatment may be increasingly used.
Author contributions Conception and design: GF, PF, MV. Patient management: GF, PF, LW, BDF, MV, JD, CB, DB (doctors); HI (secretary); FA, SB, NL (nurses); JZ, BMM, ZA (pharmacists). Collection and assembly of data: GF, PF, MV. Data analysis and interpretation: GF, PF, JD, CB, DB, LW, BDF, MV. Manuscript writing: GF, MV.

Data availability All core data are available upon request.

\section{Declarations}

Ethical approval This study was approved by our local ethics committee (CERAPH5).

Conflicts of interest The authors declare no competing interests.

\section{References}

1. Burki TK (2020) Cancer guidelines during the COVID-19 pandemic. Lancet Oncol 21:629-630

2. Karacin C, Acar R, Bal O, et al. 'Swords and Shields' against COVID-19 for patients with cancer at 'clean' and 'pandemic' hospitals: are we ready for the second wave? Support Care Cancer. Epub ahead of print 22 January 2021. https://doi.org/10.1007/ s00520-021-06001-6

3. Terpos E, Engelhardt M, Cook G et al (2020) Management of patients with multiple myeloma in the era of COVID-19 pandemic: a consensus paper from the European Myeloma Network (EMN). Leukemia 34:2000-2011

4. Infection au nouveau Coronavirus (SARS-CoV-2), COVID-19, France et Monde, /maladies-et-traumatismes/maladies-et-infections-respiratoires/infection-a-coronavirus/articles/infectionau-nouveau-coronavirus-sars-cov-2-covid-19-france-et-monde (accessed 22 April 2020)

5. Touati M, Lamarsalle L, Moreau S et al (2016) Cost savings of home bortezomib injection in patients with multiple myeloma treated by a combination care in Outpatient Hospital and Hospital care at Home. Support Care Cancer 24:5007-5014

6. Evans JM, Qiu M, MacKinnon M et al (2016) A multi-method review of home-based chemotherapy. Eur J Cancer Care 25:883-902

7. Binder AF, Handley NR, Wilde L et al (2020) Treating Hematologic Malignancies During a Pandemic: Utilizing Telehealth and Digital Technology to Optimize Care. Front Oncol 10:1183

8. Lassalle A, Thomaré P, Fronteau C et al (2016) Home administration of bortezomib in multiple myeloma is cost-effective and is preferred by patients compared with hospital administration: results of a prospective single-center study. Ann Oncol 27:314-318

9. Mittaine-Marzac B, Bagaragaza E, Ankri J, et al. Impacts on health outcomes and on resources utilization for anticancer drugs injection at home, a complex intervention: a systematic review. Support Care Cancer. Epub ahead of print 25 March 2021. https:// doi.org/10.1007/s00520-021-06145-5

Publisher's note Springer Nature remains neutral with regard to jurisdictional claims in published maps and institutional affiliations. 